\title{
Stability characteristics of the mush affected by the solid layer below
}

\author{
Min Hsing Chang ${ }^{\mathrm{a}}$, Falin Chen ${ }^{\mathrm{b}, *}$ \\ a Department of Mechanical Engineering, Yung-Ta Institute of Technology and Commerce, Pingtung 909, Taiwan, ROC \\ ${ }^{\mathrm{b}}$ Institute of Applied Mechanics, National Taiwan University, Taipei 10764, Taiwan, ROC \\ Received 26 December 2002; accepted 23 April 2003 \\ Communicated by K. Nakajima
}

\begin{abstract}
In directional solidification of binary alloys, a dendritic mush is formed between the bulk melt above and the eutectic solid below. We investigate the convective instabilities in such a three-layer system during solidification process, which differs from previous two-layer model by the solid layer. Due to the existence of the solid layer, the mush/solid interface is allowed to deform so that the mushy layer becomes less stable while the stability of the melt is virtually unchanged. The mushy layer also becomes less stable when the latent heat of fusion generated in the mush is larger, while the stability of the melt above is not affected because the heat is transferred through the solid layer towards the cooling boundary below. Present results may help reflect the reality that the heat transferring to the cooling boundary decreases with time so that the stability of the mush will be enhanced during solidification process, and explain the discrepancy between former theoretical predictions and experimental measurements.
\end{abstract}

(C) 2003 Elsevier Science B.V. All rights reserved.

Keywords: A1. Convection; A1. Directional solidification; A1. Fluid flows; A1. Heat transfer; A2. Growth from solutions

\section{Introduction}

The directional solidification is a processing technique with which metallurgists employed to develop advanced metals of exceptionally high mechanical strength as well as high resistance to heat and fatigue [1-3]. During the solidification process, several double-diffusive convections of various stability characteristics occur either in sequence or simultaneously [4-7]. They are the double-diffusive salt-finger convection formed above the dendritic mush, the isolated plumes of

\footnotetext{
*Corresponding author. Fax: + 886-2-3639290.

E-mail address: falin@iam.ntu.edu.tw (F. Chen).
}

depleted buoyant fluid generated directly from the interior of the dendritic mush, the large-scale circulation of the bulk fluid induced by the degeneration of the salt-finger convection, and the horizontal double-diffusive layers resulted from the breakdown of the plumes at the upper surface of the melt [7]. Each convective flow can seriously affect the crystalline of the resultant castings, and under some circumstances may in turn deteriorate the mechanical properties of the products.

Due to the importance of the application and the rich phenomena resulted from the interaction between thermodynamical and fluid mechanical effects, a great research effort has been devoted to 
studying the convective flows arisen during the solidification process [8-17]. One of the important issues discussed extensively is the formation of plumes, or more precisely the mechanisms triggering the onset of plume convection. This is because that the striking analogy between the "chimneys" (corresponding to the plumes) found in the dendritic mush of aqueous ammonium chloride solutions and the freckles formed in alloy castings. The freckles are of columnar shapes vertically extending from the bottom to the top of the casting mould. Both the composition and the metallic structure of the freckle are very different from the base alloy, causing a deleterious effect on the mechanical strength of casting. Recently, theoretical analyses have indicated that the formation of plumes is a result from the convection in the mush [11,12], while all of them neglected the effect due to the existence of the solid layer below, which turns out to be influential on the stability of the mush, as will be shown in the present paper.

Up to date, in all the theoretical analyses the physical model shown in Fig. 1 has been considered, while some of them considered the combination of the fluid and the mushy layers as a whole $[8,10,13-16]$, and some focused on the mush alone $[9,11,12]$. For both approaches, it is

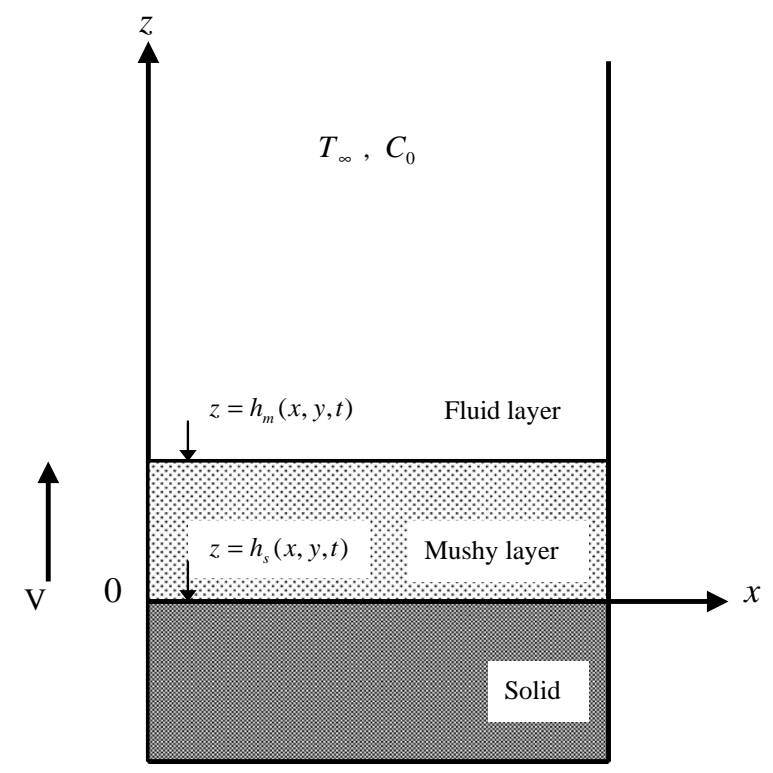

Fig. 1. Configuration of the physical system. always assumed that the mush/solid interface is of constant temperature and concentration and no deformation is allowed. In the present paper, we consider a three-layer system (see Fig. 1) in which the dendritic mush is sandwiched between the melt above and the solid layer below. This configuration fits more the reality, such as the casting mould or the experimental box that the cooling boundary with fixed temperature locates at the bottom of the solid layer. The depth of the solid increases with time, while the temperature at the mush/solid interface remains at eutectic temperature and the bottom of the solid is fixed at the preset value. As a result, the heat transferred across the solid layer decreases as a function of time, so that the heat taken from the mush to the bottom decreases with time, which would surely influence the stability of the melt in the mush and in turn the rigor of the convection of the system, and we will discuss the influence in the present paper.

The paper is organized as follows. In Section 2 we describe the physical problem and the corresponding governing equations and boundary conditions. In Section 3 we derive the basic state solution, based on which the linear stability analysis is implemented. In Section 4 the linearized small perturbation equations and associated boundary conditions are shown, the normal mode analysis is applied, and a shooting technique is employed to solve the resultant eigenvalue problem. In Section 5 the stability characteristics of the system are discussed, special attention is paid to the influence of the deformable mush/solid interface as well as the heat transferred through the solid layer. And finally in Section 6 concluding remarks are drawn.

\section{Problem description and mathematical model}

Consider the three-layer system shown in Fig. 1: A binary solution of concentration $C_{0}$ and temperature $T_{\infty}$ is solidified from below. The mushy layer is sandwiched between the melt above and the eutectic solid below, extending from the mush/solid interface $z=h_{\mathrm{s}}(x, y, t)$ to the melt/ mush interface $z=h_{\mathrm{m}}(x, y, t)$, where $x$ and $y$ are the horizontal axes of Cartesian coordinate and $t$ is 
the time. Assumptions made for the present model are as follows: Both the interfaces move upwards with a constant velocity $V$, the temperature at $h_{\mathrm{s}}$ is fixed at the eutectic temperature $T_{\mathrm{E}}, C_{0}$ is greater than the eutectic concentration $C_{\mathrm{E}}, T_{\infty}$ is higher than the liquidus temperature $T_{\mathrm{L}}\left(C_{0}\right)$, the dendritic mush is in thermodynamic equilibrium so that the temperature and concentration of the mush satisfy the liquidus relation

$T=T_{\mathrm{L}}\left(C_{0}\right)+\Gamma\left(C-C_{0}\right)$,

where $\Gamma$ is the slope of the liquidus and is assumed to be constant. The density of the fluid is written as

$\rho=\rho_{0}\left[1+\beta\left(C-C_{0}\right)\right]$,

where $\rho_{0}$ is the reference density and $\beta=\beta^{*}-\Gamma \alpha^{*}$, $\alpha^{*}$ and $\beta^{*}$ are the thermal and solute expansion coefficients, respectively.

The following scales are used to render the governing equations dimensionless: velocity by $V$, length by $H$ where $H=\kappa / V$, time by $\kappa / V^{2}$, and pressure by $\rho_{0} g \beta \Delta C \kappa / V$. In the above scales, $\kappa$ is the thermal diffusivity, $\Delta C$ is the compositional scale given by $C_{0}-C_{\mathrm{E}}$, and $g$ is the gravitational acceleration. The non-dimensional temperature and concentration can be written as

$\theta=\frac{T-T_{\mathrm{L}}\left(C_{0}\right)}{\Delta T}, \quad \Theta=\frac{C-C_{0}}{\Delta C}$,

where $\Delta T=\Gamma \Delta C=T_{\mathrm{L}}\left(C_{0}\right)-T_{\mathrm{E}}$. We employ Galilean transformation to attach the coordinates on the mush/solid interface $h_{\mathrm{s}}$ and obtain the nondimensional governing equations and boundary conditions. Note that the present mathematical formulations are similar to that considered by Worster [8] and are essentially the same with that of Chen et al. [10] except that these two papers did not take the solid layer into account. Worster [8] considered only the cases where the thermal field does not interact with the solutal field because he assumed the buoyancy ratio $A$ (see the definition below Eq. (27)) to be zero. For the convenience of subsequent discussions and the completeness of the mathematical presentation, nonetheless, we show in the following the complete set of equations and boundary conditions. In the fluid layer $z>h_{\mathrm{m}}(x, y, t)$ we have

$\nabla \cdot \mathbf{U}=0$,

$$
\begin{aligned}
& \frac{1}{\sigma}\left(\frac{\partial}{\partial t}-\frac{\partial}{\partial z}+\mathbf{U} \cdot \nabla\right) \mathbf{U}= \nabla^{2} \mathbf{U}+\left(R_{\mathrm{T}} \theta-R_{\mathrm{C}} \Theta\right) \mathbf{e}_{\mathrm{z}} \\
&-R_{\mathrm{m}} \phi \nabla p \\
&\left(\frac{\partial}{\partial t}-\frac{\partial}{\partial z}+\mathbf{U} \cdot \nabla\right) \theta=\nabla^{2} \theta \\
&\left(\frac{\partial}{\partial t}-\frac{\partial}{\partial z}+\mathbf{U} \cdot \nabla\right) \Theta=\varepsilon \nabla^{2} \Theta
\end{aligned}
$$

Eqs. (4a)-(4d) are the continuity equation and the equations of conservation of momentum, heat and solute, respectively. In these equations, $U$ is the velocity, $\sigma=v / \kappa$ is the Prandtl number, $v$ is the kinematic viscosity, $p$ is the pressure and $\varepsilon=D_{1} / \kappa$ is the Lewis number. The thermal and solute Rayleigh numbers are given by

$R_{\mathrm{T}}=\frac{g \alpha^{*} H^{3} \Delta T}{\kappa v}, \quad R_{\mathrm{C}}=\frac{g \beta^{*} H^{3} \Delta C}{\kappa v}$.

In the mushy layer $h_{\mathrm{s}}(x, y, t)<z<h_{\mathrm{m}}(x, y, t)$ we have

$\nabla \cdot \mathbf{U}=0$,

$\left(\frac{\partial}{\partial t}-\frac{\partial}{\partial z}+\mathbf{U} \cdot \nabla\right) \theta+\varphi\left(\frac{\partial}{\partial t}-\frac{\partial}{\partial z}\right) \chi=\nabla^{2} \theta$,

$\left(\frac{\partial}{\partial t}-\frac{\partial}{\partial z}\right)[\chi(\theta-\xi)]+\mathbf{U} \cdot \nabla \theta=0$,

$\frac{\mathbf{U}}{\Pi(\chi)}=-R_{\mathrm{m}}\left(\nabla p+\theta \mathbf{e}_{\mathrm{z}}\right)$.

Eqs. (5a)-(5d) are respectively the continuity equation, the energy conservation equation, the solute conservation equation, and Darcy equation. Note that the material properties of the solid and liquid phases are assumed to be identical in the mush. Besides, the solute diffusion is neglected because the solute diffusivity is very small, and the Boussinesq approximation is applied. The Stefan number $\varphi$, the concentration ratio $\xi$, and the Rayleigh number $R_{\mathrm{m}}$ are defined respectively as

$\varphi=\frac{L}{c \Delta T}, \quad \xi=\frac{C_{\mathrm{s}}-C_{0}}{\Delta C}, \quad R_{\mathrm{m}}=\frac{g \beta \Pi^{*} H \Delta C}{\kappa v}$,

where $L$ is the latent heat per unit volume, $c$ the specific heat per unit volume, and $C_{\mathrm{s}}$ the solid 
concentration. The Rayleigh number $R_{\mathrm{m}}$ measures the ratio between the destabilizing compositional buoyancy and the stabilizing thermal diffusion, being a major parameter accounting for the stability of the system. Moreover, $\Pi(\chi)$ is the permeability, which is a function of porosity $\chi[10]$, $\phi=H^{2} / \Pi^{*}$ is the inverse Darcy number and $\Pi^{*}$ the reference permeability. Finally, in the solid layer $z<h_{s}(x, y, t)$, we have the conservation of energy given by

$$
\left(\frac{\partial}{\partial t}-\frac{\partial}{\partial z}\right) \theta=\nabla^{2} \theta
$$

The boundary conditions at infinite far field are that both the temperature and concentration are assumed to remain the same as the original solution. Moreover, the height of the tank is assumed large enough that we can neglect the influence due to the possible deformation of the free surface on the convective flow occurring mostly in the vicinity of the melt/mush interface. Accordingly, as $z \rightarrow \infty$ we have

$\theta \rightarrow \theta_{\infty}, \quad \Theta \rightarrow 0, \quad \mathbf{U} \rightarrow \mathbf{0}$.

$(7 a-c)$

At the melt/mush interface $z=h_{\mathrm{m}}$ the boundary conditions are

$$
\begin{aligned}
& \theta=\Theta, \quad \mathbf{n} \cdot \nabla \theta=\mathbf{n} \cdot \nabla \Theta, \quad[\mathbf{n} \cdot \mathbf{U}]=0, \\
& {[\theta]=0, \quad[\mathbf{n} \cdot \nabla \theta]=0,} \\
& \chi=1, \quad[p]=0, \\
& \left.\frac{\partial \mathbf{u}_{2}}{\partial n}\right|_{h_{\mathrm{m}}^{+}}=\Lambda \sqrt{\frac{\phi}{\Pi(1)}}\left(\left.\mathbf{u}_{2}\right|_{h_{\mathrm{m}}^{+}}-\left.\mathbf{u}_{2}\right|_{h_{\mathrm{m}}^{-}}\right),
\end{aligned}
$$

where [ ] denotes the jump of the enclosed quantity across the interface, and $\mathbf{n}$ is the normal vector to the interface. Eq. (8a) represents the concentration continuity, Eq. (8b)is the marginal equilibrium condition [18], Eqs. (8c) and (8d) account respectively for the mass and temperature continuities, Eq. (8e) represents the heat flux continuity, Eq. (8f) means that the porosity is fixed to be unity, Eq. (8g) denotes the normal stress continuity, and Eq. (8h) is the empirical condition proposed by Beavers and Joseph [19] describing the relation between the planar velocities $\mathbf{u}_{\mathbf{2}}$ in the fluid above and that of the mush below, where $\Lambda$ is an empirical constant determined by experiment
[19]. The boundary conditions at mush/solid interface $z=h_{\mathrm{s}}$ are

$$
\begin{aligned}
& \theta=-1, \quad \mathbf{n} \cdot \mathbf{U}=0, \\
& \mathbf{n} \cdot\left[\left.\nabla \theta\right|_{h_{\mathrm{s}}^{+}}-\left.\nabla \theta\right|_{h_{\mathrm{s}}^{-}}+\chi \varphi\left(1+\frac{\partial h_{\mathrm{s}}}{\partial t}\right) \mathbf{e}_{z}\right]=0 .(9 \mathrm{a}-\mathrm{c})
\end{aligned}
$$

Eq. (9a) is the eutectic condition fixing a relation between the temperature and the solute at the interface, so that this condition can also account for the solute conservation because the solid layer is considered to be a eutectic solid. Eq. (9b) represents the impermeable boundary that the vertical flow ceases at the interface, and Eq. (9c) accounts for the jump of heat flux caused by the freezing of the remaining melt of the mush [17].

\section{Basic state solution}

The governing Eqs. (4)-(6) and boundary conditions (7-9) admit a steady solution in which the fluid remains quiescent, i.e. $\mathbf{U}=\mathbf{0}$, and the temperature and the concentration depend only on the vertical coordinate $z$. By considering a steady and motionless situation for Eqs. (4c) and (5c), we end up with a set of second-order heat conduction equations in respectively the melt and mushy layers. These ordinary equations can then be integrated with respect to $z$, leading to the solution of exponential type when the marginal equilibrium condition Eq. (8b) is taken into account. In the fluid layer, the solutions are

$$
\begin{aligned}
& \theta_{\mathrm{b}}=\theta_{\infty}+\left(\theta_{\mathrm{i}}-\theta_{\infty}\right) \exp \left[-\left(z-h_{\mathrm{b}}\right)\right], \\
& \Theta_{\mathrm{b}}=\theta_{\mathrm{i}} \exp \left[-\left(z-h_{\mathrm{b}}\right) / \varepsilon\right],
\end{aligned}
$$

where the subscript $\mathrm{b}$ denotes the basic state, $\theta_{\mathrm{i}}$ is the temperature (or concentration) at the melt/ mush interface given by

$\theta_{\mathrm{i}}=\left(\frac{-\varepsilon}{1-\varepsilon}\right) \theta_{\infty}$

and $h_{\mathrm{b}}$ is the fixed horizontal level of the melt/ mush interface. In the mushy layer, the temperature distribution $\theta_{\mathrm{b}}$ is expressed by the implicit function

$z=\frac{\bar{\alpha}-\xi}{\bar{\alpha}-\bar{\beta}} \ln \left(\frac{\bar{\alpha}+1}{\bar{\alpha}-\theta_{\mathrm{b}}}\right)+\frac{\xi-\bar{\beta}}{\bar{\alpha}-\bar{\beta}} \ln \left(\frac{\bar{\beta}+1}{\bar{\beta}-\theta_{\mathrm{b}}}\right)$ 
and the porosity is a function of $\theta_{\mathrm{b}}$

$\chi_{\mathrm{b}}=\frac{\xi-\theta_{\mathrm{i}}}{\xi-\theta_{\mathrm{b}}}$,

where

$$
\begin{aligned}
& \bar{\alpha}=\bar{A}+\bar{B}, \quad \bar{\beta}=\bar{A}-\bar{B}, \\
& \bar{A}=\frac{1}{2}\left(\xi+\theta_{\mathrm{b}}+\varphi\right), \\
& \bar{B}=\left(\bar{A}^{2}-\theta_{\infty} \xi-\varphi \theta_{\mathrm{i}}\right)^{1 / 2} .
\end{aligned}
$$

Since the existence of the solid layer below does not influence the motionless temperature and concentration distribution in the melt and the mush, the above basic state solutions are the same with those shown in, for example, Worster [8] and Chen et al. [10]. In the solid layer, the temperature $\theta_{\mathrm{b}}$ is given by

$\theta_{\mathrm{b}}=\theta_{\infty}+\varphi-\left(1+\theta_{\infty}+\varphi\right) \exp (-z)$.

The mush/solid interface is at $z=0$ so that the height of mushy layer $h_{\mathrm{b}}$ can be obtained by substituting $\theta_{\mathrm{b}}-\theta_{\mathrm{i}}$ into Eq. (11a).

A typical result corresponding to the $26 \mathrm{wt} \%$ ammonium chloride solution is shown in Fig. 2. For the values of the physical parameters of this solution, the reader is referred to Ref. [10]. All



Fig. 2. The basic state of $\theta$ (dimensionless temperature) and $\Theta$ (dimensionless concentration) for the $26 \mathrm{wt} \%$ ammonium chloride solution. analyses of present paper are made on the basis of this binary solution. It is seen from Fig. 2 that the temperature gradient in the solid layer is much larger than that in the mushy layer, so that the latent heat of fusion generated in the mush can be transferred efficiently through the solid layer to the cooling boundary at bottom. Due to thermodynamic equilibrium condition applied in the mush [10], the dimensionless temperature and the dimensionless concentration are the same. In the fluid region, the solute changes dramatically within the very shallow solute boundary layer right above the melt/mush interface, while the temperature changes gradually from the melt/mush interface to $T_{\infty}$ across the much deeper thermal boundary layer.

\section{Linear stability analysis}

To investigate the linear stability of the basic state shown in the previous section, we superimpose a small disturbance on the basic solution, substitute the combination into Eqs. (4)-(6), and then neglect the quadratic terms in the usual way. Since the coefficients in the resultant disturbance equations depend only on $z$, we can apply the normal mode expansion proportional to $\exp \left[\omega t+\mathrm{i}\left(k_{x} x+k_{y} y\right)\right]$ to the small-disturbance quantities. Then the linearized small disturbance equations take the form as follows. In the fluid layer

$\left[D^{2}+(D-\omega)-\alpha^{2}\right] \hat{\theta}=\theta_{\mathrm{b}}^{\prime} \hat{w}$,

$\left[\varepsilon D^{2}+(D-\omega)-\varepsilon \alpha^{2}\right] \hat{\Theta}=\Theta_{\mathrm{b}}^{\prime} \hat{w}$

$\left(D^{2}-\alpha^{2}\right) \hat{w}=\hat{\Omega}$,

$\left[D^{2}+(D-\omega) / \sigma-\alpha^{2}\right] \hat{\Omega}=\alpha^{2}\left(R_{\mathrm{T}} \hat{\theta}-R_{\mathrm{C}} \hat{\Theta}\right)$,

where $D$ and the prime represent the differentiation $\mathrm{d} / \mathrm{d} z, \omega$ is the frequency of the normal mode, $\alpha=\left(k_{x}^{2}+k_{y}^{2}\right)^{1 / 2}$ is the horizontal wavenumber, $\hat{\theta}$ the disturbance temperature, $\hat{w}$ the disturbance vertical velocity, $\hat{\Theta}$ the disturbance concentration, and $\hat{\Omega}$ the disturbance vorticity. In the mushy 
layer,

$$
\begin{aligned}
& {\left[D^{2}+(D-\omega)-\alpha^{2}\right] \hat{\theta}+\varphi(D-\omega) \hat{\chi}=\theta_{\mathrm{b}}^{\prime} \hat{w}} \\
& {\left[\chi_{\mathrm{b}}(D-\omega)+\chi_{\mathrm{b}}^{\prime}\right] \hat{\theta}+\left[\left(\theta_{\mathrm{b}}-\xi\right)(D-\omega)\right.} \\
& \left.\quad+\theta_{\mathrm{b}}^{\prime}\right] \hat{\chi}=\theta_{\mathrm{b}}^{\prime} \hat{\omega} \\
& {\left[D^{2}-\frac{\Pi^{\prime}\left(\chi_{\mathrm{b}}\right)\left(\xi-\theta_{\mathrm{i}}\right)}{\Pi\left(\chi_{\mathrm{b}}\right)\left(\xi-\theta_{\mathrm{b}}\right)^{2}} \theta_{\mathrm{b}}^{\prime} D-\alpha^{2}\right] \hat{w}} \\
& \quad=\alpha^{2} \Pi\left(\chi_{\mathrm{b}}\right) R_{\mathrm{m}} \hat{\theta}
\end{aligned}
$$

where $\hat{\chi}$ is the disturbance porosity. In the solid layer,

$\left[D^{2}+(D-\omega)-\alpha^{2}\right] \hat{\theta}=0$.

Eqs. (13)-(20) are subject to the linearized boundary conditions as follows. At $z \rightarrow \infty$,

$\hat{\theta}=0, \quad \hat{\Theta}=0, \quad \hat{w}=0, \quad D \hat{w}=0$.

$(21 \mathrm{a}-\mathrm{d})$

At $z=h_{\mathrm{b}}$,

$\hat{\theta}=\hat{\Theta}, \quad D \hat{\Theta}-D \hat{\theta}=\frac{1-\varepsilon}{\varepsilon} \theta_{\mathrm{b}}^{\prime} \hat{\eta}_{1}, \quad[\hat{w}]=0$,

$(22 \mathrm{a}-\mathrm{c})$

$[\hat{\theta}]=0, \quad[D \hat{\theta}]=\frac{\varphi}{\theta_{\mathrm{i}}-\xi} \theta_{\mathrm{b}}^{\prime} \hat{\eta}_{1}$,

$\hat{\chi}=\frac{\theta_{\mathrm{b}}^{\prime}}{\theta_{\mathrm{i}}-\xi} \hat{\eta}_{1}$,

$$
\left.D^{2} \hat{w}\right|_{h_{\mathrm{b}}^{+}}=\Lambda\left(\frac{\varphi}{\Pi(1)}\right)^{1 / 2}\left(\left.D \hat{w}\right|_{h_{\mathrm{b}}^{+}}-\left.D \hat{w}\right|_{h_{\mathrm{b}}^{-}}\right)
$$

$$
\begin{aligned}
\left.D \hat{w}\right|_{h_{\mathrm{b}}^{-}}= & -\frac{\Pi(1)}{\varphi} \\
& \times\left[D \hat{\Omega}+\frac{1}{\sigma}\left(\hat{\Omega}-\omega D \hat{w}+\alpha^{2} \hat{w}\right)\right. \\
& \left.-2 \alpha^{2} D \hat{w}\right]_{h_{\mathrm{b}}^{+}}
\end{aligned}
$$

where $\hat{\eta}_{1}$ is the disturbance amplitude of $\eta_{1}$, and $\eta_{1}$ is the perturbation to the position of the melt/ mush interface, i.e. $h_{\mathrm{m}}(x, y, t)=h_{\mathrm{b}}+\eta_{1}(x, y, t)$. Similarly, we perturb the mush/solid interface $h_{\mathrm{s}}(x, y, t)=\eta_{2}(x, y, t)$, where $\eta_{2}$ is the perturbation to this interface. Then the boundary conditions at

$$
\begin{aligned}
& z=0 \text { are } \\
& \hat{w}=0, \quad\left(\frac{\left.\mathrm{d} \theta_{\mathrm{b}}\right|_{+}}{\mathrm{d} z}\right) \hat{\eta}_{2}+\left.\hat{\theta}\right|_{+}=0, \\
& \left(\frac{\left.\mathrm{d} \theta_{\mathrm{b}}\right|_{-}}{\mathrm{d} z}\right) \hat{\eta}_{2}+\left.\hat{\theta}\right|_{-}=0, \\
& \left(\frac{\left.\mathrm{d}^{2} \theta_{\mathrm{b}}\right|_{+}}{\mathrm{d} z^{2}}-\frac{\left.\mathrm{d}^{2} \theta_{\mathrm{b}}\right|_{-}}{\mathrm{d} z^{2}}+\varphi \frac{\left.\mathrm{d} \chi_{\mathrm{b}}\right|_{+}}{\mathrm{d} z}\right) \hat{\eta}_{2} \\
& \quad+\frac{\left.\mathrm{d} \hat{\theta}\right|_{+}}{\mathrm{d} z}-\frac{\left.\mathrm{d} \hat{\theta}\right|_{-}}{\mathrm{d} z}+\varphi\left(\left.\omega \chi_{\mathrm{b}}\right|_{+} \hat{\eta}_{2}+\left.\hat{\chi}\right|_{+}\right)=0,
\end{aligned}
$$

where $\hat{\eta}_{2}$ is the disturbance amplitude of $\eta_{2}$. Assume that the disturbance of temperature at $z \rightarrow-\infty$ is negligible (i.e. $\hat{\theta}=0$ ), the disturbance temperature $\hat{\theta}$ in the solid layer can be solved from Eq. (20) with the boundary condition (23c) and can be written as

$$
\begin{aligned}
\hat{\theta}= & -\left(1+\theta_{\infty}+\varphi\right) \hat{\eta}_{2} \\
& \times \exp \left\{\left[-\frac{1}{2}+a^{1 / 2} \exp \left(\frac{i \psi}{2}\right)\right] z\right\},
\end{aligned}
$$

where

$a=\left[\left(\frac{1}{4}+\alpha^{2}+\omega_{\mathrm{r}}\right)^{2}+\omega_{\mathrm{i}}^{2}\right]^{1 / 2}$,

$\cos \psi=\left(\frac{1}{4}+\alpha^{2}+\omega_{\mathrm{r}}\right) / a, \quad \sin \psi=\omega_{\mathrm{i}} / a, \quad(25 \mathrm{~b}, \mathrm{c})$ and $\omega_{\mathrm{r}}, \omega_{\mathrm{i}}$ are respectively the real and imaginary part of $\omega$. The disturbance amplitude $\hat{\eta}_{2}$ can be determined by Eq. (23d). Eqs. (13)-(19) with the boundary conditions (21)-(23) consist of a complex eigenvalue problem

$F=F\left(R_{\mathrm{m}}, \alpha, \omega ; \quad \theta_{\infty}, \varphi, \xi, \phi, \varepsilon, \sigma, A\right)$.

Note that $R_{\mathrm{T}}, R_{\mathrm{C}}$ and $R_{\mathrm{m}}$ satisfy the relations

$R_{\mathrm{T}}=A \phi R_{\mathrm{m}}, \quad R_{\mathrm{C}}=(1+A) \phi R_{\mathrm{m}}$,

where $A=\Gamma \alpha^{*} / \beta$ is the buoyancy ratio. We solve this eigenvalue problem by the shooting technique [10]. The integration is carried out in a sufficiently large truncated domain, from $z=0$ to $z=z_{\mathrm{u}} \approx 30$, so that the integrated results are independent of the value of $z_{u}$. The integration starts from the melt/mush interface $\left(z=h_{\mathrm{b}}\right)$ and ends respectively at $z_{\mathrm{u}}$ and 0 . To avoid the loss of linear independence of integration, an orthonormalization process is 
implemented by utilizing the superposition method developed by Keller [21]. An iteration procedure developed by Powell [22] is employed to seek the convergency of the eigenvalues $R_{\mathrm{m}}$ and $\omega$.

\section{System stability characteristics and discussion}

To examine the stability of the present threelayer system, we consider first the cases of Chen et al. [10] and compare the present results with their Fig. 1. Results are shown in Fig. 3, in which four cases of different buoyancy ratios $A=$ $0,0.1,0.5$, and 0.75 are considered. The results of Ref. [10] are accounted for by dashed curves and the present results by solid curves. To read this figure and those in Fig. 4, it is recalled that the instability of the present system is bimodal $[8,10]$, see for example Fig. 3(a). The local minimum of smaller wavenumber accounts for the onset of the mush-layer mode, a flow circulates across the
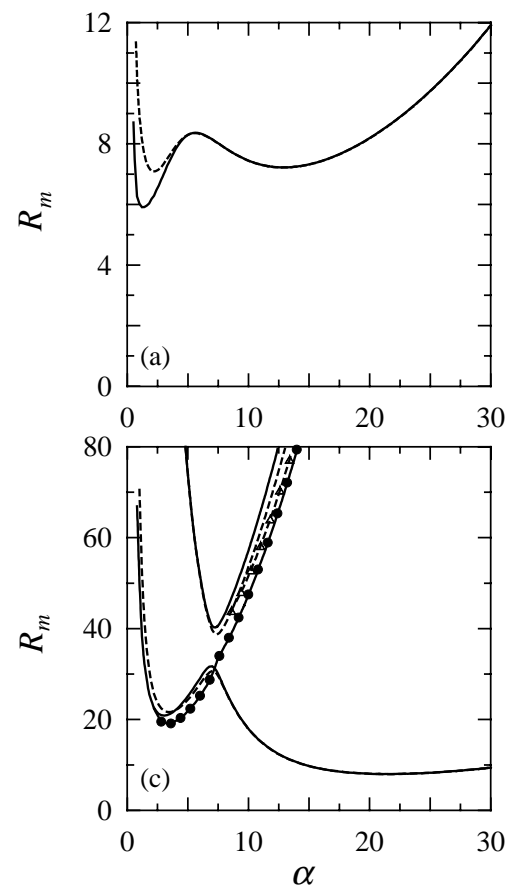

depth of mush. The local minimum of larger wavenumber accounts for the onset of the boundary-layer mode, a flow circulates within the shallow solute boundary layer above the melt/ mush interface. In general, the boundary-layer mode is less stable than the mush-layer mode, as usually found in experiments and in Figs. 3(b)-(d) as well. The boundary-layer mode will develop into the nonlinear salt-finger convection as solidification proceeds.

Results of Fig. 3 indicate that as the solid layer is considered, both the mush-layer and the oscillatory modes of the present three-layer configuration are less stable than those of previous two-layer system, while the stability of boundarylayer mode is virtually unchanged. The similar result happens to all the four cases of different buoyancy ratio $A$ considered, implying that the above conclusion applies well to different alloys. The cause that the mushy-layer and oscillatory modes become less stable is mainly due to the
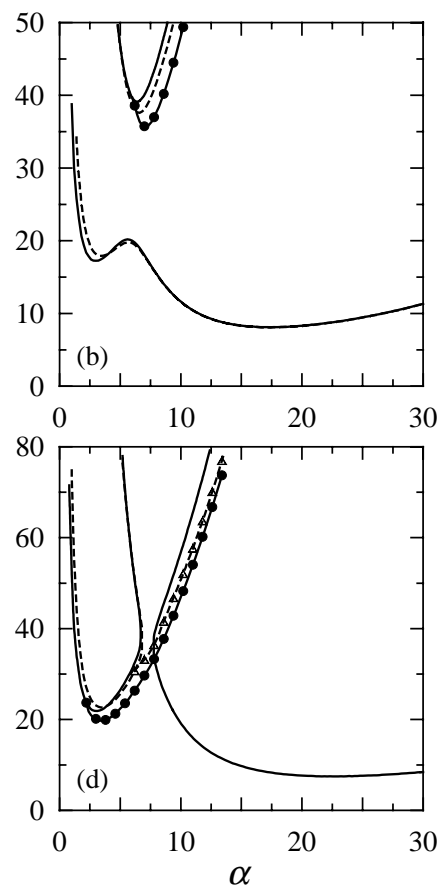

Fig. 3. The neutral curves of different instability modes for four different buoyancy ratio (a) $A=0$, (b) $A=0.1$, (c) $A=0.5$, (d) $A=0.75$. The solid curves are the results of present study and the dashed curves are those of previous study without considering the solid layer [10]. The curve with solid-circle accounts for the oscillatory mode of present three-layer system and that with blank-triangle $\triangle$ represents the oscillatory mode of previous two-layer system [10]. In the present case, $\varphi=\xi=\theta_{\infty}=1, \sigma=10, \phi=10^{5}, \varepsilon=0.025$. 

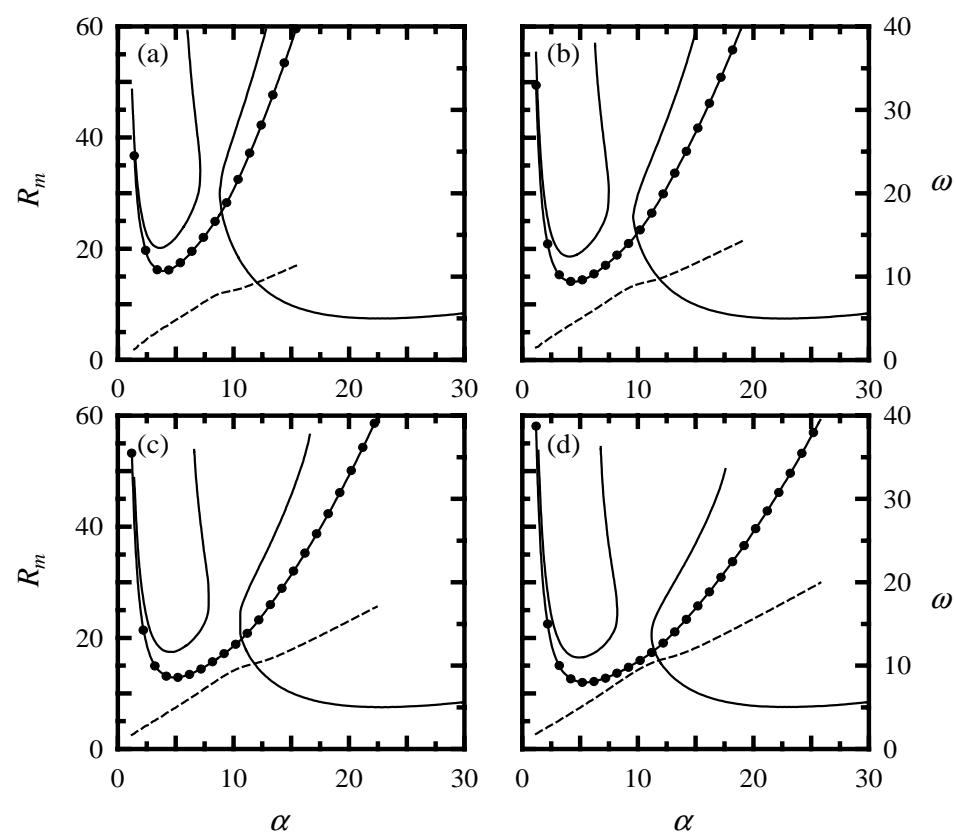

Fig. 4. Variations of neutral curves with respect to the Stephan number: (a) $\varphi=2$, (b) $\varphi=3$, (c) $\varphi=4$, (d) $\varphi=5$, where $\varphi$ is the Stephan number. The other parameters are the same as those in Fig. 3 and the buoyancy ratio $A$ is 0.75 . The curve with solid-circles represents the oscillatory mode and the dashed curve represents the corresponding oscillatory frequency $\omega$. The neutral curves of the mushy-layer mode and the oscillatory mode move downwards slowly with the increase of $\varphi$ and the corresponding critical wavenumber $\alpha_{c}$ moves rightwards. While the neutral curve of the boundary-layer mode is virtually unaffected by varying $\varphi$.

deformation of the mush/solid interface. Since both the mushy-layer and the oscillatory modes are the flows circulating mostly in the mushy layer, they accordingly become less stable as the mush/solid interface is allowed to deform. But the stability of boundary-layer mode is virtually uninfluenced because it circulates mostly in the melt, which is away from the mush/solid interface.

We note that in the experiments of directional solidification [5], the melt is solidified from below due to the cooling from the bottom boundary. In such a system, both the mush and the solid layers grow unidirectionally upwards, and the growth rates of the melt/mush and the mush/solid interfaces change with time [20]. As a result, the latent heat of fusion generated in the mush changes with time, which shall be taken out of the system by the cooling applied at the bottom boundary so that the heat transfer through the solid layer shall also change with time. More precisely speaking, since the temperatures at both the mush/solid interface and the bottom boundary are fixed whereas the thickness of the solid layer increases with time, the heat transfer rate through the solid layer to the cooling bottom boundary shall decrease with time. This unsteady effect, however, cannot be considered directly in the present investigation because we have assumed that both the interfaces move with the same constant velocity $V$ so that the latent heat generated in the mush and the heat transfer through the solid layer shall also be constant.

On the other hand, however, this unsteady effect due to the change of heat transfer through the solid layer can be investigated through the study considering the change of the Stefan number $\varphi$. According to the definition shown in (5e), the Stefan number is defined as the ratio between the latent heat of fusion and the heat capacity of 
the mushy layer. Since the height of the mushy layer as well as the temperature difference across the mushy layer are assumed to be constant in the present paper, the heat capacity of mush is accordingly constant, so that a larger Stefan number simply means a larger latent heat of fusion. This latent heat generated in the mush shall be taken out of the system by the cooling applied at the bottom. Since the temperature is fixed at the cooling boundary, a larger latent heat of mush (or a larger Stefan number) shall mean a larger heat transfer across the solid layer.

Figs. 4(a)-(d) illustrate the neutral curves of four different Stefan numbers: 2, 3, 4 and 5, respectively. The neutral curves of the mush-layer mode and the boundary-layer mode are accounted for by the solid curve, the oscillatory mode is represented by the curve with solid circles, and its frequency $\omega$ is represented by the dashed curve. It is seen that the boundary-layer mode is generally less stable than the other two modes, tending to occur first and to develop into the nonlinear saltfingers observed in experiments. As $\varphi$ increases, both the mushy-layer and the oscillatory modes become less stable while the boundary-layer mode is virtually uninfluenced. This is because the heat, either generated or transferred, occurs only in the mush so that only the flow in the mush is influenced by changing $\varphi$. We note also that the $\omega$ does not change significantly with $\varphi$ neither, implying that the speed of the traveling wave, a dynamic form the oscillatory mode appears, does not vary significantly with $\varphi$.

The above results are supported by the experiments of, for example, Chen and Chen [5]. A larger $\varphi$ means a larger heat transfer from the solid layer, or equivalently a smaller height of the solid layer, which shall occur in the beginning of the experiment. Accordingly, the system shall become more stable in the experiment as time goes on since the depth of the solid layer increases with time. In other words, the destabilizing effect resulted from the consideration of the solid layer shall become less significant as time marches. This is consistent with the observation of Chen and Chen [5] that, after some time into the experiment, the doublediffusive convection decays with time and eventually disappears.

\section{Concluding remarks}

We have investigated the effects due to the existence of the solid layer on the convective instabilities in the directionally solidifying binary solution. It is found that, because of the deformation of the mush/solid interface, the mushy-layer and the oscillatory modes, both circulate within the mush, become less stable while the stability of the boundary-layer mode circulating above the mush is virtually uninfluenced. The heat transfer through the solid layer, which can be accounted for by the Stefan number, also influences on the stabilities of the mushy-layer and the oscillatory modes while, again, cast virtually no effect on the stability of the boundary-layer mode. As a result, the stabilities of both the mushy-layer mode and the oscillatory mode are reduced as the heat transfer increases. Due to the fact that in the solidification process the depth of the solid layer increases with time so that the heat transfer through the solid layer decreases with time, present results suggest that the mush shall become more stable as solidification proceeds. This is consistent with the experimental observation [5] that, as the cooling temperature at the bottom is higher or as the heat transfer rate through the solid layer to the bottom is lower, the mush becomes more stable so that the plume convection is harder to form. This also explains partly why it usually takes a longer time to solidify the melt of the single crystal turbine blade in which no single freckle is allowed to form [1] because a smaller heat transfer rate shall be applied.The fact that a larger heat transfer through the solid layer makes the mush less stable may also help explain the discrepancy between the plume threshold conditions resulted from respectively the experimental measurements [23] and the theoretical predictions $[8,11,12]$. In the experiments, Tait et al. [23] conducted a series of experiments for aqueous ammonium chloride solutions of various viscosity so that they were able to measure the critical condition of the onset of plumes. They also found that the onset of plumes is accompanied with a mushy-layer mode convection of a hexagonal planform, with down-flow at the center and upflow along the perimeters of the hexagon. In the theoretical investigations $[8,11,12]$, in which the 
weakly nonlinear analysis for the onset of plume convection was made for the mush alone, the critical conditions were generally found to be "much" larger than the experimental data. Although some typical reasons causing this discrepancy had been discussed [12], mostly focused on the theoretical models which are believed to be different to some extent from the physical reality while partly focused on the mathematical approaches which under some circumstances may not lead to a converged solution, the present results may also shed light on another possibility that the heat transferred through the solid layer to the bottom of the experimental box may also be one of the reasons can lead to a lower critical condition of the plume threshold so that the discrepancy between experimental measurements and theoretical predictions can be smaller.

\section{Acknowledgements}

The financial support for this research through the research grants NSC 90-2212-E-002-202 and NSC 91-2212-E-132-004 of National Science Council of Taiwan is gratefully acknowledged.

\section{References}

[1] M.C. Flemings, G.E. Nereo, G. E. Trans. Metall. Soc. AIME 239 (1967) 1449.
[2] R. Mehrabian, M. Keane, M.C. Flemings, Metall. Trans. 1 (1970) 1209.

[3] M. McLean, Directionally Solidified Materials for High Temperature Service, The Metals Society, London, 1983.

[4] S.M. Copley, A.F. Giamei, S.M. Johnson, M.F. Hornbecker, Metall. Trans. 1 (1970) 2193.

[5] C.F. Chen, F. Chen, J. Fluid Mech. 227 (1991) 567.

[6] S. Tait, K. Jahrling, C. Jaupart, Nature 359 (1992) 406.

[7] F. Chen, J. Crystal Growth 179 (1997) 277.

[8] M.G. Worster, J. Fluid Mech. 237 (1992) 649.

[9] G. Amgerg, G.M. Homsy, J. Fluid Mech. 252 (1993) 79.

[10] F. Chen, J.W. Lu, T.L. Yang, J. Fluid Mech. 276 (1994) 163.

[11] D.M. Anderson, M.G. Worster, J. Fluid. Mech. 302 (1995) 307.

[12] C.A. Chung, F. Chen, J. Fluid Mech. 408 (2000) 53.

[13] W.D. Bennon, F.P. Incropera, Int. J. Heat Mass Transfer 30 (1987) 2161.

[14] C. Beckmann, R. Viskanta, Physicochem. Hydrodyn. 10 (1988) 195

[15] J.C. Heinrich, S. Felicelli, P. Nandapurka, D.R. Poirier, Metall. Trans. B 20B (1989) 883.

[16] C.S. Magirl, F.P. Incropera, J. Heat Transfer ASME Trans. 115 (1983) 1036.

[17] D.E. Loper, P.H. Roberts, Stud. Appl. Math. 106 (2001) 187.

[18] M.G. Worster, J. Fluid Mech. 167 (1986) 481.

[19] G.S. Beavers, D.D. Joseph, J. Fluid Mech. 30 (1967) 197.

[20] F. Chen, T.LY ang, J.W. Lu, J. Appl. Phys. 74 (1993) 7531.

[21] H.B. Keller, Numerical Solutions of Two Points Boundary Value Problems, SIAM, Philadelphia, MA, 1976.

[22] M.J.D. Powell, Numerical Methods for Nonlinear Algebraic Equations, in: P.H. Rabinowitz (Ed.), Gordon \& Breach, London, 1970.

[23] S. Tait, K. Jahrling, C. Jaupart, Nature 359 (1992) 406. 